\title{
Repurposing phosphodiesterase-5 inhibitors as chemoadjuvants
}

\author{
Amit K. Tiwari ${ }^{1 *}$ and Zhe-Sheng Chen ${ }^{2}$ \\ ${ }^{1}$ Department of Biomedical Sciences, College of Veterinary Medicine, Nursing and Allied Health, Tuskegee University, Tuskegee, AL, USA \\ 2 Department of Pharmaceutical Sciences, College of Pharmacy and Health Sciences, St. John's University, Queens, NY, USA \\ ${ }^{*}$ Correspondence: atiwari@mytu.tuskegee.edu
}

Edited by:

George Tegos, University of New Mexico, USA

Reviewed by:

Dominique Perez, University of New Mexico Health Sciences Center, USA

Partha Krishnamurthy, University of Kansas Medical Center, USA

Phosphodiesterase-5 (PDE5) inhibitors have shown a beneficial effect in a variety of clinical conditions, such as benign prostate hyperplasia, pulmonary arterial hypertension, female sexual arousal disorder, overactive bladder, and incontinence, Raynaud's disease, heart failure and stroke among others (Sandner et al., 2007). Three PDE5 inhibitors, sildenafil (Viagra $^{\mathrm{TM}}$ ), tadalafil (Cialis ${ }^{\mathrm{TM}}$ ) and vardenafil (Levitra ${ }^{\mathrm{TM}}$ ) are clinically approved and are widely used for the treatment of erectile dysfunction. A retrospective analysis determined that men treated with PDE5 for ED had less chance of having prostate cancer. This population of men had significantly lower documented diagnosis of elevated prostate-specific antigen and higher percentage of benign prostatic hyperplasia compared to men not treated with PDE-5 inhibitors (Chavez et al., 2013). Emerging evidence indicates that PDE5 inhibitors are multi-targeting agents and have promising results in the treatment of variety of tumors. Here we propose the possibility of repurposing of PDE5 inhibitors for adjuvant chemotherapy.

PDE5 contributes to the regulation of intracellular cyclic GMP (cGMP) pools (see Figure 1) that have been shown to be decreased along with protein kinase-G (PKG), a downstream effector of cGMP, in variety of different tumors such as breast cancer, colon cancer and human oral squamous cell carcinoma (hOSCC) (Spoto et al., 2003; Zhu and Strada, 2007; Di et al., 2010). PDE5 hydrolyzes the $3^{\prime}, 5^{\prime}$-phosphodiester bond in the second messenger molecule cGMP to biologically inactive $5^{\prime}$-GMP. There is an incomplete understanding of how PDE5 inhibitors act in cancer, yet there are reports of increased apoptosis in different tumor cell types following treatment with PDE5 inhibitors. Possible mechanisms of these anticancer effects via PDE5 inhibition mediated caspase-dependent apoptosis and cell growth arrest may be linked to concomitant increases in regulation of downstream pathways through increased cGMP-PKG levels and subsequent effects on, (1) activation of c-Jun NH2-terminal kinase (JNK), especially JNK1 pathways via phosphorylation of mitogen-activated protein kinase kinase kinase 1 (MEKK1) (Bender and Beavo, 2006), (2) decreased Wnt/ $\beta$-catenin expression and down-regulation of cyclin D1 (Thompson et al., 2000; Li et al., 2002; Tinsley et al., 2011), (3) inhibition of extracellular-signal regulated kinases $1 / 2$ (ERK1/2) and alterations in the regulation of p42/p44 mitogen activated-protein kinase (MAPK) and p21 pathways (Hou et al., 2006; Das et al., 2008). Increased PDE5 expression was shown to play a role in tumorigenesis in variety of cancers, such as non-small cell lung cancer, urinary bladder cancer, metastatic breast cancer and development of hOSCC (Piazza et al., 2001; Pusztai et al., 2003; Whitehead et al., 2003). Thus, it is hypothesized that inhibiting PDE5 activity may produce antineoplastic actions. There is a small amount of supporting data. Indeed, PDE5 inhibitors, sildenafil and vardenafil induced caspase-dependent apoptosis of B-cell chronic lymphocytic leukemia cells (Sarfati et al., 2003), whereas cytotoxic and growth suppressive effects in various breast cancer, prostate, and colon cells were seen with non-specific PDE5 inhibitors sulindac sulfone and its analogs
(Thompson et al., 2000; Piazza et al., 2001; Whitehead et al., 2003; Tinsley et al., 2011). Interestingly, PDE5 inhibitors were shown to alter the tumor microenvironment by augmenting endogenous antitumor immunity by reducing myeloidderived suppressor cell function (Serafini et al., 2006).

The accumulating body of evidence suggests that PDE5 inhibitors could interfere with the efflux functions of the $A B C$ transporters, thus sensitizing cancer cells toward cytotoxic agents that are substrates of ABC transporters (Ding et al., 2011; Shi et al., 2011; Chen et al., 2012) (see Figure 1). For example, cGMP is a substrate of $\mathrm{ABCC} 4$ and $\mathrm{ABCC} 5$ transporters that are involved in reducing its intracellular concentrations. Sildenafil reverses this phenomenon through a dual action, first it inhibits PDE5, and secondly it could block the transport function of ABCC4 and $\mathrm{ABCC}$, thus increasing the intracellular cGMP concentrations (Jedlitschky et al., 2000; Chen et al., 2001). More recently our group reported that specific PDE5 inhibitors could block the function of $\mathrm{ABC}$ transporters at clinically achievable concentrations (Ding et al., 2011; Shi et al., 2011; Chen et al., 2012). We showed that sildenafil could block the efflux functions of $\mathrm{ABCB} 1$ and $\mathrm{ABCG} 2$ transporters in cancer cells, and thus, significantly reversed the MDR-mediated efflux of substrate anticancer drugs, such as mitoxantrone, paclitaxel, and vinca alkaloids (Shi et al., 2011). Other PDE5 inhibitors, vardenafil and tadalafil were also examined for their effect on $\mathrm{ABC}$ transporter-mediated efflux in cancer cells. It was found that vardenafil in a concentration-dependent manner, significantly potentiated the cytotoxicity 


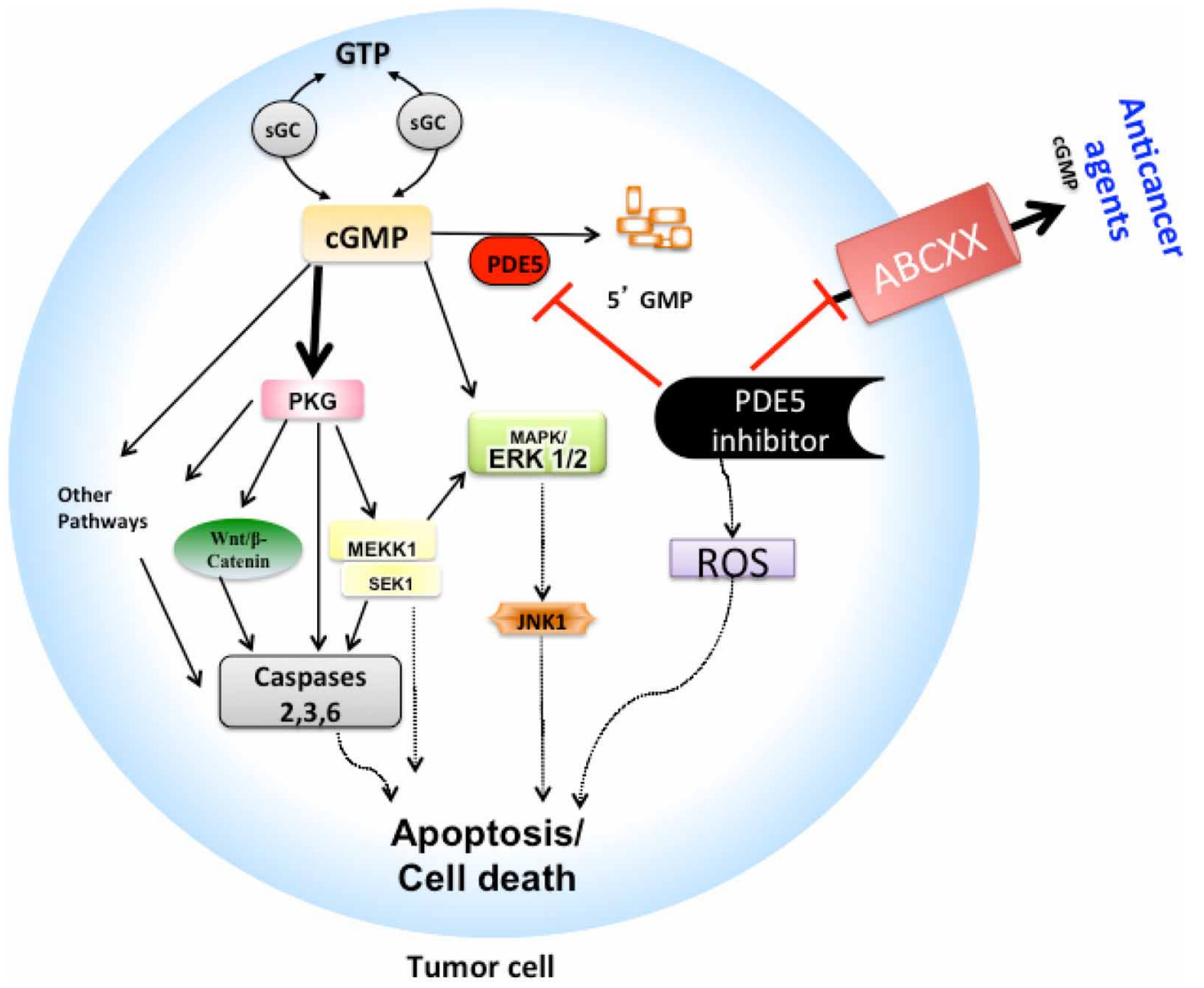

FIGURE 1 | Schematic model of PDE5 inhibitors mechanism as chemoadjuvants: Proposed model is shown (A) as how tumor cells can efflux cGMP and variety of anticancer drugs that are substrate of $A B C B 1, A B C G 2, A B C C 4, A B C C 5$, and $A B C C 10$ and so survive in the absence of PDE5 inhibitors. (B) Probable mechanism by which the PDE5 inhibitors such as sildenafil, vardenafil and tadalafil produces apoptotic activity is shown, which may be due to (1) inhibition of PDE5 activity, thus increasing CGMP-PKG activation, which leads to activation of series of signaling events including phosphorylation of $\beta$-catenin and/or

MEKK1/SEK1/JNK1 signaling pathways that eventually results in apoptosis cascade, and/or (2) inhibition of efflux function of $A B C C 4, A B C C 5$, $A B C C 10, A B C B 1$, and $A B C G 2$ drug transporters and thus increase the sensitivity of other chemotherapeutic agents that are substrates of these transporters. $A B C X X$, refers to $A B C B 1, A B C G 2, A B C C 4, A B C C 5$, or ABCC10; cGMP, cyclic guanosine monophosphate; ERK1/2, extra-cellular regulated kinases 1/2; MAPK, mitogen-activated protein kinase; PKG, Protein kinase G; PDE5, Phosphodiesterase type 5; ROS, Reactive oxygen species. of anticancer agents that are substrates of $A B C B 1$, but not that of $A B C C 1$ or ABCG2 transporters and this effect was significantly greater than that of tadalafil (Ding et al., 2011). Furthermore, sildenafil and vardenafil enhanced the activity of paclitaxel, docetaxel and vinblastine in the ABCC10-transfected HEK293 cells (Chen et al., 2012). Recently, we found that sildenafil significantly enhanced the sensitivity of specific anticancer drugs in different tumor cell lines and in ABCB1and ABCG2-bearing MDR mouse models (unpublished data). These novel functions of PDE5 inhibitors might explain why in brain tumor models, doxorubicin and herceptin transport efficacy across the bloodbrain tumor barrier was enhanced by the addition of sildenafil and vardenafil (Black et al., 2008). Combination chemotherapy with PDE5 inhibitors was shown to produce reactive oxygen species and led to apoptosis that proved to be beneficial in treatment of broad range of cancers. For example, enhanced tumor suppression and apoptotic activity was seen with a sulindac-docetaxel combination in nonsmall cell lung cancer orthotopic lung tumor model (Whitehead et al., 2003), and with a sulindac-capecitabine combination in breast cancer (Pusztai et al., 2003), and more recently, with the combination of sildenafil-doxorubicin in in vivo models of prostate cancer (Das et al., 2010). We suspect that these combination therapies may have produced enhanced anticancer activity partly due to inhibition of specific $\mathrm{ABC}$ transporters, which otherwise reduced intracellular concentration of substrate anticancer agents, but this hypothesis needs to be tested. Furthermore, since the PDE5 inhibitors are substrates of multiple ABC transporters, their individual or overlapping roles (Tiwari et al., 2013) in PDE5 disposition is an open area of research.

In summary, there is evidence that suggests that PDE5 inhibitors may have an anticancer action either by increasing cGMP-PKG and coupled downstream events or by their ability to inhibit $A B C$ transporter-mediated $\mathrm{s}$ efflux of anticancer drugs. The safety, high tolerability, and wide availability of PDE5 inhibitors 
have made this class of drug an attractive tool to investigate their role in cancer chemotherapy. Since a detailed understanding of PDE5 inhibitors as anticancer adjuvants is limited, further studies are warranted to characterize their mechanisms and establish their role.

\section{ACKNOWLEDGMENTS}

Authors are grateful to the valuable discussion and critical comments from Dr. James M. Gallo (Department of Pharmacology and System Therapeutics, Mount Sinai School of Medicine), which has made possible the commentary in the present form.

\section{REFERENCES}

Bender, A. T., and Beavo, J. A. (2006). Cyclic nucleotide phosphodiesterases: molecular regulation to clinical use. Pharmacol. Rev. 58, 488-520. doi: 10.1124/pr.58.3.5

Black, K. L., Yin, D., Ong, J. M., Hu, J., Konda, B. M., Wang, X., et al. (2008). PDE5 inhibitors enhance tumor permeability and efficacy of chemotherapy in a rat brain tumor model. Brain Res. 1230, 290-302. doi: 10.1016/j.brainres.2008.06.122

Chavez, A. H., Scott Coffield, K., Hasan Rajab, M., and Jo, C. (2013). Incidence rate of prostate cancer in men treated for erectile dysfunction with phosphodiesterase type 5 inhibitors: retrospective analysis. Asian J. Androl. 15, 246-248. doi: 10.1038/aja.2012.162

Chen, J. J., Sun, Y. L., Tiwari, A. K., Xiao, Z. J., Sodani, K., Yang, D. H., et al. (2012). PDE5 inhibitors, sildenafil and vardenafil, reverse multidrug resistance by inhibiting the efflux function of multidrug resistance protein 7 (ATPbinding Cassette C10) transporter. Cancer Sci. 103, 1531-1537. doi: 10.1111/j.1349-7006.2012. 02328.x

Chen, Z. S., Lee, K., and Kruh, G. D. (2001). Transport of cyclic nucleotides and estradiol 17beta-D-glucuronide by multidrug resistance protein 4. Resistance to 6-mercaptopurine and 6thioguanine. J. Biol. Chem. 276, 33747-33754. doi: 10.1074/jbc.M104833200

Das, A., Durrant, D., Mitchell, C., Mayton, E., Hoke, N. N., Salloum, F. N., et al. (2010). Sildenafil increases chemotherapeutic efficacy of doxorubicin in prostate cancer and ameliorates cardiac dysfunction. Proc. Natl. Acad. Sci. U.S.A. 107, 18202-18207. doi: 10.1073/pnas.1006965107

Das, A., Xi, L., and Kukreja, R. C. (2008). Protein kinase G-dependent cardioprotective mechanism of phosphodiesterase-5 inhibition involves phosphorylation of ERK and GSK3beta. J. Biol. Chem. 283, 29572-29585. doi: 10.1074/jbc.M801547200

Di, X., Gennings, C., Bear, H. D., Graham, L. J., Sheth, C. M., White, K. L., et al. (2010). Influence of the phosphodiesterase-5 inhibitor, sildenafil, on sensitivity to chemotherapy in breast tumor cells. Breast Cancer Res. Treat. 124, 349-360. doi: 10.1007/ s10549-010-0765-7

Ding, P. R., Tiwari, A. K., Ohnuma, S., Lee, J. W., An, X., Dai, C. L., et al. (2011). The phosphodiesterase5 inhibitor vardenafil is a potent inhibitor of ABCB1/P-glycoprotein transporter. PLoS ONE 6:e19329. doi: 10.1371/journal.pone.0019329

Hou, Y., Gupta, N., Schoenlein, P., Wong, E., Martindale, R., Ganapathy, V., et al. (2006). An anti-tumor role for cGMP-dependent protein kinase. Cancer Lett. 240, 60-68. doi: 10.1016/j.canlet.2005.08.035

Jedlitschky, G., Burchell, B., and Keppler, D. (2000). The multidrug resistance protein 5 functions as an ATP-dependent export pump for cyclic nucleotides. J. Biol. Chem. 275, 30069-30074. doi: 10.1074/jbc.M005463200

Li, H., Liu, L., David, M. L., Whitehead, C. M., Chen, M., Fetter, J. R., et al. (2002). Pro-apoptotic actions of exisulind and CP461 in SW480 colon tumor cells involve beta-catenin and cyclin D1 downregulation. Biochem. Pharmacol. 64, 1325-1336. doi: 10.1016/S0006-2952(02)01345-X

Piazza, G. A., Thompson, W. J., Pamukcu, R., Alila, H. W., Whitehead, C. M., Liu, L., et al. (2001). Exisulind, a novel proapoptotic drug, inhibits rat urinary bladder tumorigenesis. Cancer Res. 61, 3961-3968.

Pusztai, L., Zhen, J. H., Arun, B., Rivera, E., Whitehead, C., Thompson, W. J., et al. (2003). Phase I and II study of exisulind in combination with capecitabine in patients with metastatic breast cancer. J. Clin. Oncol. 21, 3454-3461. doi: 10.1200/JCO.2003.02.114

Sandner, P., Hutter, J., Tinel, H., Ziegelbauer, K., and Bischoff, E. (2007). PDE5 inhibitors beyond erectile dysfunction. Int. J. Impot. Res. 19, 533-543. doi: 10.1038/sj.ijir.3901577

Sarfati, M., Mateo, V., Baudet, S., Rubio, M., Fernandez, C., Davi, F., et al. (2003). Sildenafil and vardenafil, types 5 and 6 phosphodiesterase inhibitors, induce caspase-dependent apoptosis of B-chronic lymphocytic leukemia cells. Blood 101, 265-269. doi: 10.1182/blood-2002-01-0075

Serafini, P., Meckel, K., Kelso, M., Noonan, K., Califano, J., Koch, W., et al. (2006). Phosphodiesterase-5 inhibition augments endogenous antitumor immunity by reducing myeloid-derived suppressor cell function. J. Exp. Med. 203, 2691-2702. doi: 10.1084/jem.20061104
Shi, Z., Tiwari, A. K., Shukla, S., Robey, R. W., Singh, S., Kim, I. W., et al. (2011). Sildenafil reverses ABCB1- and ABCG2-mediated chemotherapeutic drug resistance. Cancer Res. 71, 3029-3041. doi: 10.1158/0008-5472.CAN-10-3820

Spoto, G., Fioroni, M., Rubini, C., Contento, A., Di Nicola, M., Forcella, S., et al. (2003). Cyclic guanosine monophosphate phosphodiesterase activity in human gingival carcinoma. J. Oral Pathol. Med. 32, 189-194. doi: 10.1034/j.1600-0714.2003.00083.x

Thompson, W. J., Piazza, G. A., Li, H., Liu, L., Fetter, J., Zhu, B., et al. (2000). Exisulind induction of apoptosis involves guanosine 3', 5'cyclic monophosphate phosphodiesterase inhibition, protein kinase $\mathrm{G}$ activation, and attenuated beta-catenin. Cancer Res. 60, 3338-3342.

Tinsley, H. N., Gary, B. D., Keeton, A. B., Lu, W., Li, Y., and Piazza, G. A. (2011). Inhibition of PDE5 by sulindac sulfide selectively induces apoptosis and attenuates oncogenic Wnt/beta-cateninmediated transcription in human breast tumor cells. Cancer Prev. Res. (Phila.) 4, 1275-1284. doi: 10.1158/1940-6207.CAPR-11-0095

Tiwari, A. K., Zhang, R., and Gallo, J. M. (2013). Overlapping functions of $\mathrm{ABC}$ transporters in topotecan disposition as determined in gene knockout mouse models. Mol. Cancer Ther. doi: 10.1158/1535-7163.MCT-13-0100. [Epub ahead of print].

Whitehead, C. M., Earle, K. A., Fetter, J., Xu, S., Hartman, T., Chan, D. C., et al. (2003). Exisulindinduced apoptosis in a non-small cell lung cancer orthotopic lung tumor model augments docetaxel treatment and contributes to increased survival. Mol. Cancer Ther. 2, 479-488.

Zhu, B., and Strada, S. J. (2007). The novel functions of cGMP-specific phosphodiesterase 5 and its inhibitors in carcinoma cells and pulmonary/cardiovascular vessels. Curr. Top. Med. Chem. 7, 437-454. doi: $10.2174 / 156802607779941198$

Received: 27 April 2013; accepted: 08 June 2013; published online: 25 June 2013.

Citation: Tiwari AK and Chen Z-S (2013) Repurposing phosphodiesterase-5 inhibitors as chemoadjuvants. Front. Pharmacol. 4:82. doi: 10.3389/fphar.2013.00082 This article was submitted to Frontiers in Experimental Pharmacology and Drug Discovery, a specialty of Frontiers in Pharmacology.

Copyright (c) 2013 Tiwari and Chen. This is an openaccess article distributed under the terms of the Creative Commons Attribution License, which permits use, distribution and reproduction in other forums, provided the original authors and source are credited and subject to any copyright notices concerning any third-party graphics etc. 